One of our patients reported that her psoriatic lesions got worse on anakinra. Forty one patients reported no adverse events during the treatment.

Conclusions: Anakinra was effective in controlling the symptoms in colchicineresistant FMF cases. It was also effective in FMF related amyloidosis. The major cause of treatment termination was injection site reactions. Anakinra seems to be an effective alternative in patients who have insufficient response to colchicine.

Disclosure of Interest: None declared

DOI: 10.1136/annrheumdis-2018-eular.7269

\section{THU0612 BONE SARCOIDOSIS: A RETROSPECTIVE MULTICENTER STUDY OF 27 CASES}

C. Glanowski ${ }^{1}$, R. Mestiri ${ }^{2}$, L. Bialé ${ }^{1}$, T. Carmoi $^{2}$, D. Lechevalier ${ }^{1}$, G. Leroux $^{3}$, D. Saadoun ${ }^{3}$, C. Chapelon-Abric ${ }^{3}$, P. Cacoub ${ }^{3} .{ }^{1}$ Rheumatology; ${ }^{2}$ Internal Medecine, Hôpital Bégin, Saint Mandé: ${ }^{3}$ Internal Medecine, Hôpital de la Pitié Salpêtrière, Paris, France

Background: Studies on bone involvement of sarcoidosis (BS) are scarse. Objectives: To analyse in depth main features, treatments and follow up of patients presenting a BS.

Methods: Among 926 patients with a proved sarcoidosis from four tertiary hospitals in Paris (France) seen between 2000 and 2015, all cases of BS were retrospectively analysed for demography, clinical features, biological tests and imaging results. Inclusion criteria were a) a bone biopsy with epithelioid granuloma and no casein necrosis, or b) radiological evidence of BS, after exclusion of other diagnoses.

Results: 27 out $926(2.9 \%)$ sarcoidosis patients fullfilled inclusion criteria for BS. Most patients were caucasian (56\%), M/F sex ratio 1.5, 30\% were active smokers, mean age at sarcoidosis diagnosis was $39 \pm 12$ years and at BS diagnosis 43 \pm 11 years. Extra-osseous involvement of sarcoidosis was found in lymph nodes $(93 \%)$, lungs $(78 \%)$, skin $(52 \%)$, CNS $(33 \%)$, ENT $(33 \%)$, and heart $(19 \%)$. BS was symptomatic in $15 / 27(56 \%)$ patients i.e. bone pain $(15 / 15)$, local inflammation (5/15), bone deformation (3/15), arthritis (4/15), and myalgia (5/15). BS was never the revealing symptom of sarcoidosis. BS was more frequently symptomatic when it was a Perthes-Jüngling osteitis and an appendicular skeletton involvement.

On imaging exams, BS lesions were found at the spine skeletton alone (14/27, $52 \%)$, appendicular skeletton alone $(10 / 27,37 \%)$ or both $(3 / 27,11 \%)$. BS lesions had an aspect of pseudo-metastasis (59\%), micro-cysts (Perthes-Jungling, 37\%) or Paget disease (4\%). Bone lesion was unique in $22 \%$ and $26 \%$ of patients had more than 10 lesions. When a bone biopsy was done it always confirmed the diagnosis $(n=9)$; in all other cases extra-osseous biopsies confirmed the diagnosis of sarcoidosis.

Nine patients received a treatment for BS, i.e. prednisone $(n=8,0.25-1 \mathrm{mg} / \mathrm{kg} /$ day), hydroxychloroquine $(n=8)$, and methotrexate $(n=5)$. Response to treatment was complete $(n=3)$, partial $(n=4)$ or nul $(n=2)$. Of note, 21 out of 27 patients received an immunosuppressant for a severe form of systemic sarcoidosis $(n=11)$ or for a steroid-sparing effect $(n=10)$. A relapse of BS was noted in 13 patients, with a mean number of relapse of $2 .^{1-24}$ After a mean follow up of 49 months, BS was in complete remission $(8 / 27,30 \%)$, partial remission $(16 / 27,59 \%)$ or remained active $(3 / 27,11 \%)$.

Conclusions: Bone involvement remains a rare manifestation of sarcoidosis. It was symptomatic in $56 \%$ of patients, mainly when Perthes-Jüngling osteitis and appendicular skeletton involvement were present. Extra-osseous involvement of sarcoidosis were always present at the time of BS diagnosis. Treatment remains difficult with frequent relapses.

Disclosure of Interest: None declared

DOI: 10.1136/annrheumdis-2018-eular.1143

\section{THU0613 THE FREQUENCY AND CHARACTERISTICS OF HEADACHE IN BEHCET'S DISEASE AND ITS EVALUATION BY TRANSCRANIAL DOPPLER ULTRASONOGRAPHY}

F. Salgür ${ }^{1}$, D. Üsküdar Cansu ${ }^{2}$, N. Uzuner ${ }^{3}, \underline{\text { C. Korkmaz }}{ }^{2} .{ }^{1}$ Internal Medicine, Endocrinology, Kent Hospital, Izmir, ${ }^{2}$ Internal Medicine, Rheumatology; ${ }^{3}$ Neurology, Eskişehir Osmangazi University, Eskişehir, Turkey

Background: Behcet's disease (BD) is a multisystem vasculitic disease and the most often neurologic manifestation of BD is headache. Transcranial Doppler ultrasonography (TCD) is a test which used for evaluating the changes in blood flow velocity developed against visual stimulation. It is not well known TCD findings in BD patients who suffered from headache.

Objectives: To evaluate the frequency and the types of headache and to investigate cerebral reactivity by TCD in BD patients.

Methods: 113 patients with BD diagnosed based on diagnostic criteria of BD by ISG and 40 healthy individuals were included in the study. The patients with BD who had neurological involvement were not included to the study. Headache type was specified by a specialist neurologist according to International classification of headache disorders society criteria. TCD was applied to 62 patients with BD and 40 healthy individuals. TCD results were evaluated by a specialist neurologist.

Results: Headache was determined in $89(78.8 \%)$ patients with BD. It was statistically significant compared to $\mathrm{HC}$ group $(60 \%, \mathrm{p}<0.03) .48$ of $89 \mathrm{BD}$ patients had tension type of headache and 33 of them had migrainous type. No significant difference was found between BD patients and $\mathrm{HC}$ group in terms of cerebral reactivity by TCD. Low pulsatility index for both the right side and the left side were noted in BD patients suffering from headache compared to BD patients not having headache $(p<0.006, p<0.003)$. No significant differences were found between tension type and migrainous type of headache in terms of TCD parameters.

Conclusions: Headache is common in BD patients, but cerebral reactivity is maintained.

Acknowledgements: None

Disclosure of Interest: None declared

DOI: 10.1136/annrheumdis-2018-eular.6396

\section{THU0614 INTERSTITIAL LUNG DISEASE IN PATIENTS WITH ANTISYNTHETASE SYNDROME AND ANTI-R052 ANTIBODIES POSITIVE}

C. Aguilera $\operatorname{Cros}^{1}$, A. Ruiz Román ${ }^{1}$, L. Méndez Díaz¹, G. Morote lbarrola², N. Garrido Puñal ${ }^{1}$, M. Arcila Durán ${ }^{1}$, M.A. Montes Cano ${ }^{3}$, J.A. Rodriguez Portal ${ }^{4}$. ${ }^{1}$ Rheumatology, University Hospital Virgen del Rocío; ${ }^{2}$ Rheumatology, Quirón Sacred Heart Hospital; ${ }^{3}$ Immunology; ${ }^{4}$ Pneumology, University Hospital Virgen del Rocío, Seville, Spain

Background: Antisynthetase syndrome (ASS) is characterised by the presence of myositis, arthritis, interstitial lung disease (ILD), fever, Raynaud's phenomenon and mechanical's hand, in the presence of antisynthetase autoantibodies (AA), the most frequent being anti-Jo1, anti-PL7 and anti-PL12. An association between ASS and anti-Ro52 with increased ILD has been described and it is believed that the presence of both antibodies is accompanied by a more severe ILD.

Objectives: To describe the clinical and analytical characteristics of a cohort of patients with ASS. To analyse the lung involvement in this type of patient and to determine the possible relationship between the different subtypes of ILD and the presence of anti-Ro52.

Methods: Retrospective descriptive study of patients treated in our Hospital (2006-2017), with AA and at least 2 clinical characteristics. The data was obtained through the review of medical records.

Variables analysed:age, sex, age, smoking, clinical presentation, diagnosis of ASS, associated neoplasia and paraneoplastic syndrome (PS)(neoplasia 3 years before or after the diagnosis of ASS), muscle enzymes (CK and aldolase), autoimmunity, glucocorticoids (GC), immunosuppressants (IS), diagnosis of ILD, HRCT pattern (High Resolution Computed Tomography) and respiratory function tests (RFT) at the beginning of ILD.

Results: We included 27 patients (20 women), mean age $61 \pm 13$ years. $7.4 \%$ smokers and $18.5 \%$ ex-smokers. $88.8 \%$ were anti-Jo1,7.4\% anti-PL12\% and $3.7 \%$ anti-PL7. Anti-Ro52 present in 18 patients. The most common clinical presentation:ILD $88 \%$ (59\% had Ro52), followed by myositis $85 \%$ (40\% are dermatomyositis), arthritis $81 \%$, mechanic's hand $51 \%$, fever $37 \%$ and Raynaud's phenomenon 25\%. The classic triad (arthritis, myositis, ILD) was present in 16 patients. Three patients presented neoplasia in the course of the disease, being identified as PS. Elevation of CK in $70 \%$ and aldolase in $74 \%$. $96 \%$ of patients have been treated with GC and IS.

The HRCT patterns were:non-specific interstitial pneumonia (NSIP)(66\%), usual interstitial pneumonia (UIP) (29\%),organised cryptogenic pneumonia (OP)(4\%) baseline RFT were performed in 19 patients. Diagnosis of ASS and ILD, both entities appear at the same time in 6 patients, in 3 patients the ILD appears before and in 14 after. In these, the median duration (range) of the ASS until the diagnosis of ILD was 1 year (0-1).

There is no relationship between the HRCT and anti-Ro52 patterns (chi-square considering the exact distribution $\mathrm{p}=0.892$ ), nor between the ILD and anti-Ro52 (Fisher exact test $\mathrm{p}=0.999$ ).

Conclusions: Our results, in general, agree with what is published in the literature. Three patients have an uncommon presentation of ASS, with a diagnosis of ILD prior to myopathy (in most of the published cases, myositis precedes or coincides with the onset of IDL), and it is important to include ASS in the differential diagnosis of ILD. In our cohort, the association between ILD and anti-Ro52 has not been demonstrated, nor among the different subtypes of ILD to Ro52. Therefore, prospective studies with a greater number of patients are necessary to define Ro52's role in the development of ILD in ASS.

Disclosure of Interest: None declared

DOI: 10.1136/annrheumdis-2018-eular.3146 